FTUV/94-43

IFIC/94-38

\title{
The tau weak-magnetic dipole moment
}

\author{
J. Bernabéu, G.A. González-Sprinberg, M.Tung and J. Vidal \\ Departament de Física Teòrica, Universitat de València, \\ and IFIC, Centre Mixt Univ. Valencia-CSIC \\ E-46100 Burjassot (València), Spain \\ August 1994
}

\begin{abstract}
We calculate the prediction for the anomalous weak-magnetic form factor of the tau lepton at $q^{2}=M_{Z}^{2}$ within the Standard Model. With all particles on-shell, this is a electroweak gauge invariant quantity. Its value is $a_{\tau}^{w}\left(M_{Z}^{2}\right)=-(2.10+0.61 i) \times 10^{-6}$. We show that the transverse and normal components of the single-tau polarization of tau pairs produced at $e^{+} e^{-}$ unpolarized collisions are sensitive to the real and absorptive parts of the anomalous weak-magnetic dipole moment of the tau. The sensitivity one can achieve at LEP in the measurement of this dipole moment is discussed.
\end{abstract}




\section{Introduction}

The anomalous magnetic dipole moments of the electron and muon provide very precise tests of quantum electrodynamics. The Standard Model predictions can also be confronted with these properties. In particular, $\tau$-physics still offers an open window to surprises.

In this paper we calculate, within the Standard Model, the one loop anomalous weakmagnetic moment (AWMM) of the $\tau$ lepton at the energy scale of the $Z$, and find a way to measure it. We show that for $e^{+} e^{-} \longrightarrow \tau^{+} \tau^{-}$unpolarized scattering at the $Z$-peak, the transverse (within the collision plane) and normal (to the collision plane) single $\tau$ polarizations are very sensitive to the real and imaginary parts of the anomalous weak-magnetic $\left(a_{\tau}^{w}\left(M_{Z}^{2}\right)\right)$ dipole form factor, respectively. We discuss our results as a background in order to separate this signal in the search for new physics.

Polarization measurements are accessible for the $\tau$ by means of the energy and angular distribution of its decay products. The angular distribution of the $\tau$-polarization, measured at LEP [1], contains separate information [2, 3, 4] on both the average polarization, measuring parity violation in the $Z-\tau^{+}-\tau^{-}$vertex, and the $Z$-polarization, measuring parity violation in the $Z-e^{+}-e^{-}$vertex. The information on the $Z-e^{+}-e^{-}$vertex is also available from the cross section asymmetry for longitudinally polarized beams [5], as measured by SLD. This allows a test of neutral current universality and, within the Standard Model, a precise determination of $\sin ^{2} \theta_{w}$. No other component of the single $\tau$-polarization is allowed in the Standard Model for unpolarized beams, in the limit of zero-mass fermions.

For the $e^{+} e^{-} \longrightarrow \tau^{+} \tau^{-}$process at the $Z$-peak, the spin density matrix of the produced $\tau$ pairs has single $\tau$-polarization terms that translate into the energy and angular distribution of the decay products. One can try to isolate a weak-magnetic dipole moment term, looking for observables sensitive to this property in the spin density matrix of the $\tau$ pairs. This kind of studies was done by the Heidelberg group [6, 7, 8] in order to isolate the real and absorptive parts of a weak-electric dipole moment from spin correlations in 
$e^{+} e^{-} \longrightarrow \tau^{+} \tau^{-}$decay products. The $\tau$ weak-magnetic moment was also investigated in [9] by looking to forward and backward transverse asymmetries.

In Ref. [10] it has been demonstrated that the single transverse and normal $\tau$ polarization terms contain separate information about the (real part of the) weak-magnetic and weak-electric dipole moments, respectively.

In this paper, with the help of the angular distribution of the hadronic decay products acting as analyzers of the spin components [11, 12, 13, 14], we explicitly construct asymmetries that can be used as observables in the measurement of the anomalous weakmagnetic form factor at $q^{2}=M_{Z}^{2}$. In particular, we show how the transverse and normal components of the single $\tau$-polarization provide information about the real and imaginary parts of the weak-magnetic form factor $a_{\tau}^{w}\left(M_{Z}^{2}\right)$.

In section 2 we calculate the Standard Model prediction for the one loop correction to the $\tau$ anomalous weak-magnetic form factor at $q^{2}=M_{Z}^{2}$. This magnitude measures the weak-magnetic coupling $Z-\tau-\tau$. We show that its value is essentially determined by the ratio of the square of the fermion mass to the weak-boson mass. Furthermore, it is governed by both kinds of diagrams present and not present in the photon vertex case. A very tiny dependence on the Higgs mass is obtained.

In section 3 we show that $a_{\tau}^{w}\left(M_{Z}^{2}\right)$ can be measured by using the transverse and normal polarization of single $\tau$ 's. We extend our previous study on the dipole moments of Ref.[10], so as to include the absorptive part of AWMM. We find that the normal polarization of single $\tau$ 's is sensitive to the absorptive part of AWMM. Finally, we discuss the sensitivity that can be achieved at LEP in the measurement of this form factor. The appendix contains some of the definitions and formulas we have used in the computation.

\section{Anomalous weak-magnetic moment}

In this section we calculate the Standard Model prediction for the anomalous weakmagnetic form factor $\left(a_{\tau}^{w}\left(M_{Z}^{2}\right)\right)$ of the $\tau$ lepton at $q^{2}=M_{Z}^{2}$. This is an order $\alpha$ electroweak radiative correction to the weak magnetic moment. The matrix element of 
the vector neutral current coupled to the $Z$ is written, using Lorentz covariance, in the form

$$
\bar{u}\left(p_{-}\right) V^{\mu}\left(p_{-}, p_{+}\right) v\left(p_{+}\right)=\bar{u}\left(p_{-}\right)\left[\frac{v\left(q^{2}\right) \gamma^{\mu}}{2 s_{w} c_{w}}+i \frac{a_{\tau}^{w}\left(q^{2}\right)}{2 m_{\tau}} \sigma^{\mu \eta} q_{\eta}\right] v\left(p_{+}\right)
$$

where $q=p_{-}+p_{+}$, $e$ is the proton charge and $s_{w}, c_{w}$ are the weak mixing angle sine and cosine, respectively. The first term $v\left(q^{2}\right)$ is the Dirac vertex (or charge radius) form factor and it is present at tree level with a value $v\left(q^{2}\right)=\frac{1}{2}-2 s_{w}^{2}$, whereas the second form factor only appears due to quantum corrections. Only the on-shell vertex with $q^{2}=M_{Z}^{2}$ is entitled to be electroweak gauge invariant in the Standard Model. $a_{\tau}^{w}$ can have contributions from both new physics or electroweak radiative corrections to the Standard Model. We calculate the leading Standard Model contribution to $a_{\tau}^{w}$. Oneloop contributions are formally of order $\alpha$, but the magnitude of each diagram is in fact also governed by the weak-boson or Higgs mass-factors like $\frac{m_{\tau}^{2}}{M_{Z}^{2}}$ or $\frac{m_{\tau}^{2}}{M_{\Phi}^{2}}$. We compute
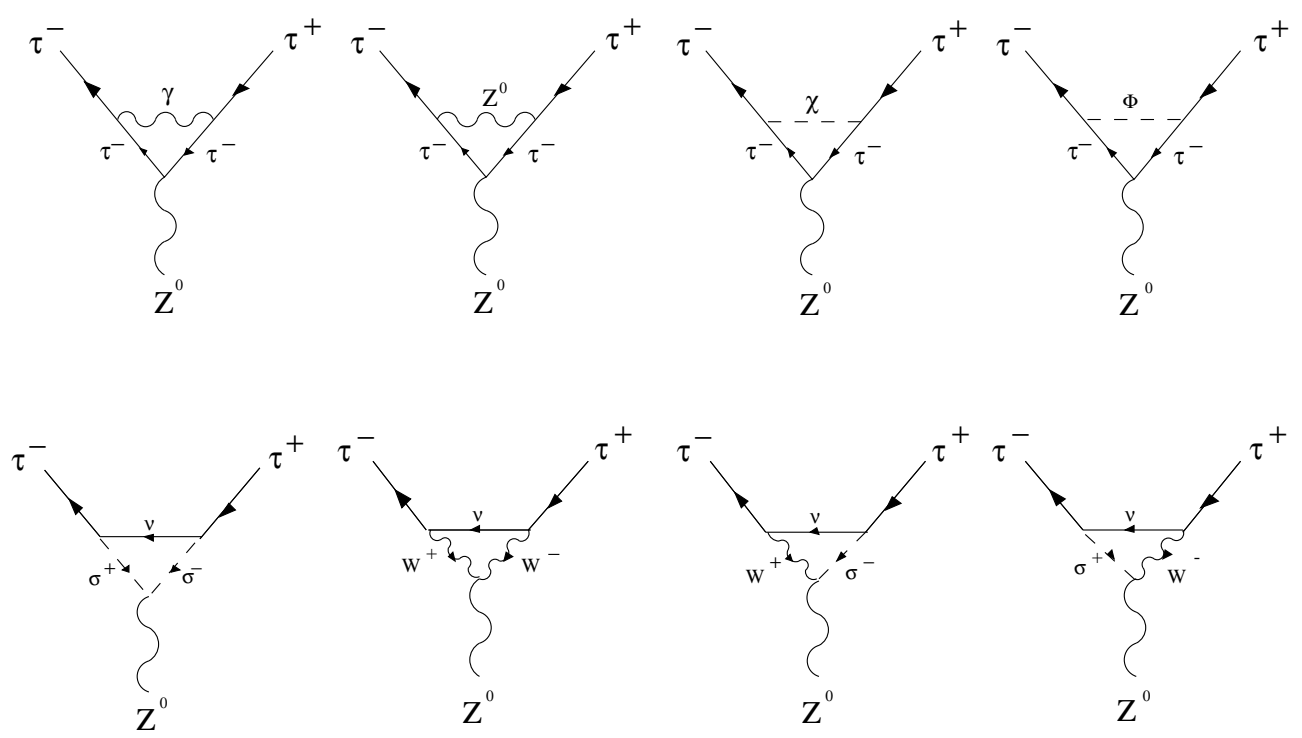

Figure 1: Contributing Feynman diagrams to $a_{\tau}^{w}$ in the t'Hooft-Feynman gauge that are also present for the anomalous magnetic moment (photon vertex) in the electroweak theory.

the AWMM in the t'Hooft-Feynman gauge, where no ambiguities in the finite parts are 
present [15]. In principle, there are 14 diagrams to compute, 6 of which are not present in the photon vertex case. The eight diagrams that have a photon analogue are shown in figure 1, and the new ones are shown in figure 2. All contributions are written as:
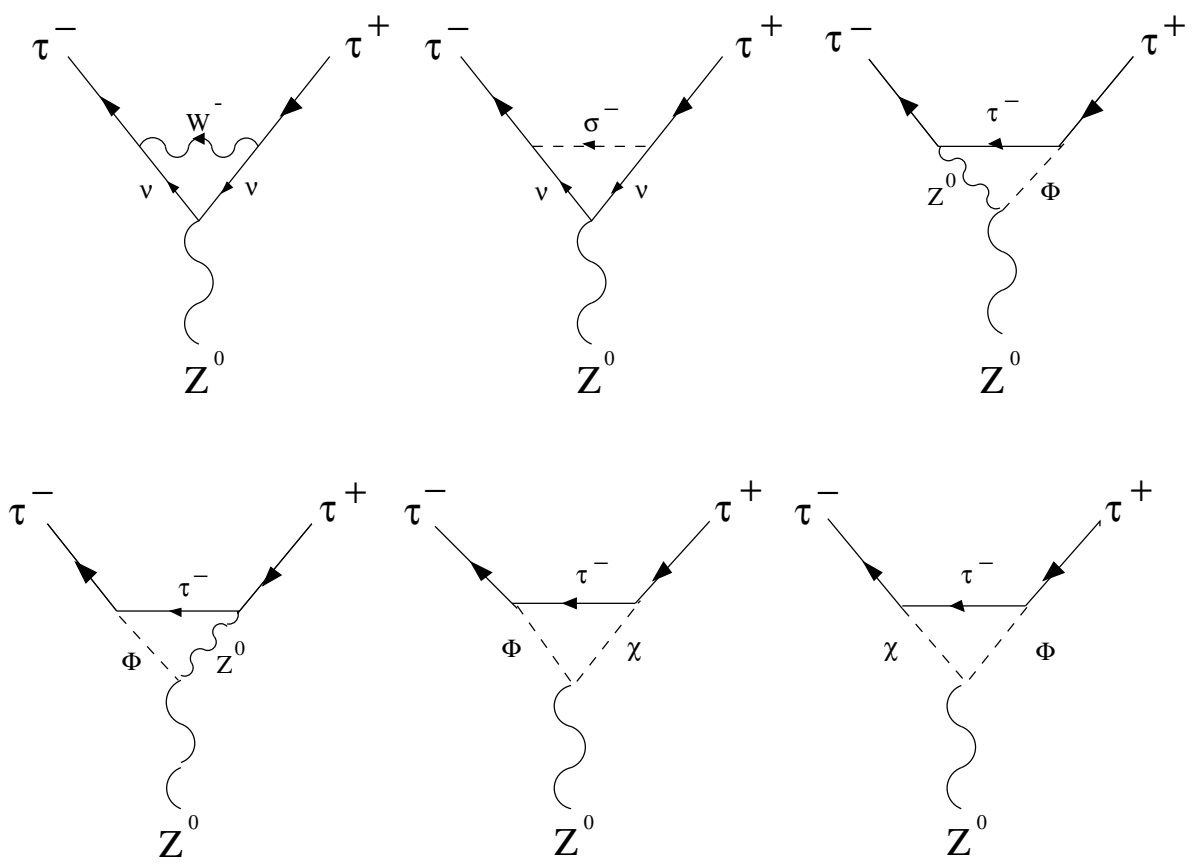

Figure 2: Contributing Feynman diagrams to $a_{\tau}^{w}$ in the t'Hooft-Feynman gauge that are not present in the anomalous magnetic moment case.

$$
a_{A B C}=\frac{\alpha}{4 \pi} \frac{m_{\tau}^{2}}{M_{Z}^{2}} \sum_{i j} c_{i j} I_{i j}{ }^{A B C}
$$

where $A, B$ and $C$ are the particles circulating in the loop, counting clockwise in the diagrams from the particle between the two fermion lines, $c_{i j}$ are coefficients, and $I_{i j}{ }^{A B C} \equiv I_{i j}\left(m_{\tau}^{2}, q^{2}, m_{\tau}^{2}, m_{A}^{2}, m_{B}^{2}, m_{C}^{2}\right)$ are scalar, vector or tensor functions defined in Eqs. A.4) and (A.5) of the Appendix.

When computing the diagrams we only select the tensor structure related to the AWMM (which is finite and needs no renormalization), and we also verify the vector current conservation as a check of our expressions (there is no induced $\left(p_{-}+p /\right)^{\mu}$ term in Eq.(1)). The external lines are on the mass shell, i.e. $p_{-}^{2}=m_{\tau}^{2}, p_{+}^{2}=m_{\tau}^{2}$ and 
$\left(p_{-}+p_{+}\right)^{2}=M_{Z}^{2}$ respectively. We denote by $\sigma^{ \pm}$the charged non-physical Higgs and by $\chi$ and $\Phi$ the neutral non-physical and physical ones. Some of the diagrams with the propagation of Higgs or would-be Goldstone bosons particles are suppressed by extra $\left(\frac{m_{\tau}^{2}}{M_{Z, \Phi}^{2}}\right)$ terms in such a way that the $a_{\chi \tau \tau}, a_{\Phi \tau \tau}, a_{\sigma \nu \nu}$ and $a_{\sigma \sigma \nu}$ contributions to $a_{\tau}^{w}$ are negligible. Diagrams in which the Higgs and the neutral would-be Goldstone boson particles couple to the $Z$ only contribute to the axial form factor and not to the magnetic moment $\left(a_{\tau \Phi \chi}=a_{\tau \chi \Phi}=0\right)$. In the following we give the different leading contributions, where the notation is self-explanatory:

$$
\begin{aligned}
& a_{\gamma \tau \tau}=\frac{\alpha}{4 \pi} \frac{m_{\tau}^{2}}{M_{Z}^{2}} \frac{4 v M_{Z}^{2}}{s_{w} c_{w}}\left[I_{10}+I_{22}-I_{21}\right]^{\gamma \tau \tau} \\
& a_{Z \tau \tau}=\frac{\alpha}{4 \pi} \frac{m_{\tau}^{2}}{M_{Z}^{2}} \frac{v M_{Z}^{2}}{s_{w}^{3} c_{w}^{3}}\left[-4 a^{2} I_{00}+\left(v^{2}+11 a^{2}\right) I_{10}+\left(v^{2}+3 a^{2}\right)\left(I_{22}-I_{21}\right)\right] z_{\tau \tau} \\
& a_{\nu W W}=\frac{\alpha}{4 \pi} \frac{m_{\tau}^{2}}{M_{Z}^{2}} \frac{M_{W}^{2}}{s_{w}^{3} c_{w}}\left[I_{10}+2 I_{21}-2 I_{22}\right]^{\nu W W} \\
& a_{\nu W \sigma}=-\frac{\alpha}{4 \pi} \frac{m_{\tau}^{2}}{M_{Z}^{2}} \frac{M_{Z}^{2}}{2 s_{w} c_{w}} I_{10}{ }^{\nu W \sigma} \\
& a_{\nu \sigma W}=-\frac{\alpha}{4 \pi} \frac{m_{\tau}^{2}}{M_{Z}^{2}} \frac{M_{Z}^{2}}{2 s_{w} c_{w}} I_{10}^{\nu \sigma W} \\
& a_{\tau \Phi Z}=-\frac{\alpha}{4 \pi} \frac{m_{\tau}^{2}}{M_{Z}^{2}} \frac{v M_{Z}^{2}}{2 s_{w}^{3} c_{w}^{3}} I_{10}{ }^{\tau \Phi Z} \\
& a_{W \nu \nu}=\frac{\alpha}{4 \pi} \frac{m_{\tau}^{2}}{M_{Z}^{2}} \frac{M_{W}^{2}}{s_{w}^{3} c_{w}^{3}}\left[I_{00}-3 I_{10}-I_{22}+I_{21}\right] W \nu \nu \\
& a_{\tau Z \Phi}=-\frac{m_{\tau}^{2}}{M_{Z}^{2}} \frac{v M_{Z}^{2}}{2 s_{w}^{3} c_{w}^{3}} I_{10}{ }^{\tau Z \Phi}
\end{aligned}
$$

where $v=-\frac{1}{2}+2 s_{w}^{2}$ and $a=-\frac{1}{2}$ are the vector and axial vector $Z-\tau^{+}-\tau^{-}$couplings. The order of magnitude of each diagram is given basically by the weak boson mass scale: all are of the same order given by $\frac{\alpha}{4 \pi} \frac{m_{\tau}^{2}}{M_{Z}^{2}}$. 
The $I_{i j}{ }^{A B C}$ functions were analytically computed in terms of dilogarithm functions, and checked with a numerical integration for the $m_{\tau} \rightarrow 0$ limit. Some details are given in the Appendix. We then obtain that the numerical contribution of each diagram is:

$$
\begin{aligned}
& a_{\gamma \tau \tau}=-\frac{\alpha}{4 \pi} \frac{m_{\tau}^{2}}{M_{Z}^{2}}(1.32-0.52 i) \simeq(3.12-1.23 i) \times 10^{-7} \\
& a_{Z \tau \tau}=\frac{\alpha}{4 \pi} \frac{m_{\tau}^{2}}{M_{Z}^{2}}(0.17+0.08 i) \simeq(3.92+1.88 i) \times 10^{-8} \\
& a_{\nu W W}=\frac{\alpha}{4 \pi} \frac{m_{\tau}^{2}}{M_{Z}^{2}}(-7.07) \simeq-1.68 \times 10^{-6} \\
& a_{\nu W \sigma}=\frac{\alpha}{4 \pi} \frac{m_{\tau}^{2}}{M_{Z}^{2}} 0.45 \simeq 1.06 \times 10^{-7} \\
& a_{\nu \sigma W}=\frac{\alpha}{4 \pi} \frac{m_{\tau}^{2}}{M_{Z}^{2}} 0.45 \simeq 1.06 \times 10^{-7} \\
& a_{\tau \Phi Z}=a_{\tau Z \Phi}=-\frac{\alpha}{4 \pi} \frac{m_{\tau}^{2}}{M_{Z}^{2}}(0.07 ; 0.03 ; 0.02) \simeq-(0.15 ; 0.07 ; 0.04) \times 10^{-7} \\
& a_{W \nu \nu}=\frac{\alpha}{4 \pi} \frac{m_{\tau}^{2}}{M_{Z}^{2}}(-4.11-2.12 i) \simeq-(0.974+0.502 i) \times 10^{-6}
\end{aligned}
$$

where the values between parenthesis for $a_{\tau \Phi Z}=a_{\tau Z \Phi} \quad$ correspond to $\frac{M_{\Phi}}{M_{Z}}=1,2,3$ respectively.

Finally, the value of the computed AWMM is

$$
a_{\tau}^{w}\left(M_{Z}^{2}\right)=-(2.10+0.61 i) \times 10^{-6}
$$

The Higgs mass only modifies the real part of this result less than a $1 \%$, from the value $-2.12 \times 10^{-6}$ to $-2.10 \times 10^{-6}$ for $1<\frac{M_{\Phi}}{M_{Z}}<3$, and in Eq.(5) we have chosen $M_{\Phi}=2 M_{Z}$.

To our knowledge, no other calculation of this value has been reported up to now. In Ref.16] a formal presentation of Feynman amplitudes in the unitary gauge is done, but no explicit computation is made. There it is argued that the procedure would be legitimate if a rigorous computation in a renormalizable gauge, as we have done, is made. 
We observe that the form factor has an absorptive part of the same order as the real one. We should point out that, contrary to the well known electroweak anomalous magnetic moment, this absorptive part is due to the fact that we compute on the $Z$ mass shell $q^{2}=M_{Z}^{2}$, not $q^{2}=0$. In fact, one expects a non-vanishing imaginary part coming from unitarity. We have also shown that the main contribution comes from the diagrams with $W$-exchange. Finally we would like to stress that this on shell result is gauge invariant, as this form factor is a linearly independent Lorentz matrix part of the contribution to the physical $Z \longrightarrow \tau^{+} \tau^{-}$decay.

\section{Observables related to the AWMM}

In this section we show that the $a_{\tau}^{w}$ form factor can be measured by observables related to the single $\tau$ polarization. Keeping only up to linear terms in the spin and in the weak dipole moments and neglecting terms proportional to the electron mass, the tree level $e^{+} e^{-} \longrightarrow \tau^{+} \tau^{-}$cross section at the $Z$-peak can be written as:

$$
\frac{d \sigma}{d \Omega_{\tau^{-}}}=\frac{d \sigma^{0}}{d \Omega_{\tau^{-}}}+\frac{d \sigma^{S}}{d \Omega_{\tau^{-}}}
$$

where the first term collects the spin independent terms, whereas the second one takes into account the linear terms in the spin:

$$
\begin{aligned}
\frac{d \sigma^{S}}{d \Omega_{\tau^{-}}}= & \frac{\alpha^{2} \beta}{128 s_{w}^{3} c_{w}^{3}} \frac{1}{\Gamma_{Z}^{2}}\left[\left(s_{-}+s_{+}\right)_{x} X_{+}+\left(s_{-}+s_{+}\right)_{y} Y_{+}+\left(s_{-}+s_{+}\right)_{z} Z_{+}+\right. \\
& \left.\left(s_{-}-s_{+}\right)_{y} Y_{-}\right]
\end{aligned}
$$

where $s_{ \pm}$are the polarization vectors of $\tau^{ \pm}$in the proper reference frame.

In Ref. 10 it is shown that 1$)$ the transverse polarization term $\left(X_{+}\right)$is proportional to the real part of the AWMM except for a helicity-flip suppressed tree level background from the Standard Model, 2) the normal polarization term $\left(Y_{-}\right)$is proportional to the weak-electric form factor $d_{\tau}^{w}$, and 3$)$ the longitudinal polarization term $\left(Z_{+}\right)$has the well known Standard Model contribution plus a quantum correction given by the real part of 
the AWMM. The existence of an absorptive part in the weak-magnetic moment induces a new component $Y_{+}$in the normal polarization. These components are:

$$
\begin{aligned}
X_{+}= & a \sin \theta_{\tau^{-}}\left\{-\left[2 v^{2}+\left(v^{2}+a^{2}\right) \beta \cos \theta_{\tau^{-}}\right] \frac{v}{\gamma s_{w} c_{w}}+\right. \\
& \left.2 \gamma\left[2 v^{2}\left(2-\beta^{2}\right)+\left(v^{2}+a^{2}\right) \beta \cos \theta_{\tau^{-}}\right] \operatorname{Re}\left(a_{\tau}^{w}\right)\right\} \\
Y_{+}= & -2 v \gamma \beta \sin \theta_{\tau^{-}}\left[2 a^{2}+\left(v^{2}+a^{2}\right) \beta \cos \theta_{\tau^{-}}\right] \operatorname{Im}\left(a_{\tau}^{w}\right) \\
Y_{-}= & 2 a \gamma \beta \sin \theta_{\tau^{-}}\left[2 v^{2}+\left(v^{2}+a^{2}\right) \beta \cos \theta_{\tau^{-}}\right]\left(2 m_{\tau} d_{\tau}^{w} / e\right) \\
Z_{+}= & -\frac{v a}{s_{w} c_{w}}\left[\left(v^{2}+a^{2}\right) \beta\left(1+\cos ^{2} \theta_{\tau^{-}}\right)+2\left(v^{2}+\beta^{2} a^{2}\right) \cos \theta_{\tau^{-}}\right] \\
& +2 a\left[4 v^{2} \cos \theta_{\tau^{-}}+\left(v^{2}+a^{2}\right) \beta\left(1+\cos ^{2} \theta_{\tau^{-}}\right)\right] \operatorname{Re}\left(a_{\tau^{w}}^{w}\right)
\end{aligned}
$$

where $\alpha$ is the fine structure constant, $\Gamma_{Z}$ is the $Z$-width, $\gamma=\frac{M_{Z}}{2 m_{\tau}}, \beta=\left(1-\frac{1}{\gamma^{2}}\right)^{\frac{1}{2}}$ are the dilation factor and $\tau$ velocity, respectively, and $d_{\tau}^{w}$ is the weak-electric form factor. The reference frame is chosen such that the outgoing $\tau^{-}$momenta is along the $z$ axis and the incoming $e^{-}$momenta is in the $x-z$ plane, and $\theta_{\tau^{-}}$is the angle determined by these two momenta. Terms with $\left(s_{-}-s_{+}\right)_{x, y, z}$ factors in Eq.(7) carry all the information about the $\mathrm{CP}$ violating pieces of the lagrangean. The normal polarization is even under parity, then only $a \cdot v^{2} \cdot d_{\tau}^{w}$ or $a^{3} \cdot d_{\tau}^{w}$ terms are allowed in $Y_{-}$(see Eq.(10)), in contrast with the case in the spin-spin correlation observables, where the leading term is $a^{2} \cdot v \cdot d_{\tau}^{w}$. The new term with $\left(s_{-}+s_{+}\right)_{y}$ induced by the $\operatorname{Im}\left(a_{\tau}^{w}\right)$ is CP-conserving and it is a time reversal-odd observable generated by an absorptive part. The dependence with $a^{2} \cdot v \cdot a_{\tau}^{w}$ or $v^{3} \cdot a_{\tau}^{w}$ is associated with the normal polarization being even under parity. Transverse polarization of a single $\tau$ (along the $\mathrm{x}$-axis) is parity-odd and time reversal-even, and it can only arise from the interference of both helicity conserving and helicity flipping amplitudes. The first term of $X_{+}$in Eq.(8) comes from helicity flipping suppressed $\left(\frac{1}{\gamma} \equiv \frac{2 m_{\tau}}{M_{Z}}\right)$ amplitudes in the Standard Model and the second one comes from the $\gamma$-enhanced chirality flipping weak-magnetic tensorial $a_{\tau}^{w}$ vertex.

From Eqs.(6) and (可) the $e^{+} e^{-} \rightarrow \tau^{+} \tau^{-} \rightarrow h_{1}^{+} X h_{2}^{-} \nu_{\tau}$ and $h_{1}^{+} \overline{\nu_{\tau}} h_{2}^{-} X$ cross sections [3, 11 are: 


$$
\begin{gathered}
\frac{d \sigma\left(e^{+} e^{-} \rightarrow \tau^{+} \tau^{-} \rightarrow h_{1}^{+} X h_{2}^{-} \nu_{\tau}\right)}{d\left(\cos \theta_{\tau^{-}}\right) d \phi_{h_{2}^{-}}}=\operatorname{Br}\left(\tau^{-} \rightarrow h_{2}^{-} \nu_{\tau}\right) \operatorname{Br}\left(\tau^{+} \rightarrow h_{1}^{+} X\right) \times \\
{\left[4 \frac{d \sigma^{0}}{d \Omega_{\tau^{-}}}+\frac{\alpha^{2} \beta \pi}{128 s_{w}^{3} c_{w}^{3} \Gamma_{Z}^{2}} \alpha_{h_{2}^{-}}\left(X_{+} \cos \phi_{h_{2}^{-}}+\left(Y_{-}+Y_{+}\right) \sin \phi_{h_{2}^{-}}\right)\right]} \\
\frac{d \sigma\left(e^{+} e^{-} \rightarrow \tau^{+} \tau^{-} \rightarrow h_{1}^{+} \overline{\nu_{\tau}} h_{2}^{-} X\right)}{d\left(\cos \theta_{\tau^{-}}\right) d \phi_{h_{1}^{+}}}=\operatorname{Br}\left(\tau^{-} \rightarrow h_{2}^{-} X\right) \operatorname{Br}\left(\tau^{+} \rightarrow h_{1}^{+} \overline{\nu_{\tau}}\right) \times \\
{\left[4 \frac{d \sigma^{0}}{d \Omega_{\tau^{-}}}+\frac{\alpha^{2} \beta \pi}{128 s_{w}^{3} c_{w}^{3} \Gamma_{Z}^{2}} \alpha_{h_{1}^{+}}\left(-X_{+} \cos \phi_{h_{1}^{+}}+\left(Y_{-}-Y_{+}\right) \sin \phi_{h_{1}^{+}}\right)\right]}
\end{gathered}
$$

where the angle $\phi_{h}$ is the azimuthal hadron angle in the frame we have already defined. All other angles have been integrated out. The longitudinal polarization term $\left(Z_{+}\right)$ disappears when the polar angle $\theta_{h}$ of the hadron is integrated out. For $\pi$ and $\rho$ mesons the magnitude of the parameter $\alpha_{h}$ is $\alpha_{\pi}=0.97$ and $\alpha_{\rho}=0.46$. The spin correlation terms give no contribution to the angular distributions Eqs.(12) and (13).

With the $\tau$ direction fully reconstructed [17] in semileptonic decays, as shown in Ref.[10], we can get information about the AWMM, by defining the following asymmetry of the $\tau$-decay products:

$$
A_{c c}^{\mp}=\frac{\sigma_{c c}^{\mp}(+)-\sigma_{c c}^{\mp}(-)}{\sigma_{c c}^{\mp}(+)+\sigma_{c c}^{\mp}(-)}
$$

with

$$
\sigma_{c c}^{\mp}(+)=\left[\int_{0}^{1} d\left(\cos \theta_{\tau^{-}}\right) \int_{-\pi / 2}^{\pi / 2} d \phi_{h^{\mp}}+\int_{-1}^{0} d\left(\cos \theta_{\tau^{-}}\right) \int_{\pi / 2}^{\frac{3}{2} \pi} d \phi_{h^{\mp}}\right] \frac{d \sigma}{d\left(\cos \theta_{\tau^{-}}\right) d \phi_{h^{\mp}}}
$$

and

$$
\sigma_{c c}^{\mp}(-)=\left[\int_{0}^{1} d\left(\cos \theta_{\tau^{-}}\right) \int_{\pi / 2}^{\frac{3}{2} \pi} d \phi_{h^{\mp}}+\int_{-1}^{0} d\left(\cos \theta_{\tau^{-}}\right) \int_{-\pi / 2}^{\pi / 2} d \phi_{h^{\mp}}\right] \frac{d \sigma}{d\left(\cos \theta_{\tau^{-}}\right) d \phi_{h^{\mp}}}
$$

This asymmetry selects the leading $\cos \theta_{\tau^{-}} \cos \phi_{h^{\mp}}$ term of the cross section. After some algebra one finds

$$
A_{c c}^{\mp}=\mp \alpha_{h} \frac{s_{w} c_{w}}{4 \beta} \frac{v^{2}+a^{2}}{a^{3}}\left[-\frac{v}{\gamma s_{w} c_{w}}+2 \gamma \operatorname{Re}\left(a_{\tau}^{w}\right)\right]
$$

with opposite values for $\tau^{-}$and $\tau^{+}$. 
For numerical results we consider $10^{7} Z$ events and one $\tau$ decaying into $\pi \nu_{\tau}$ or $\rho \nu_{\tau}$ (i.e. $h_{1}, h_{2}=\pi$ or $\rho$ in (12) and (13) respectively), while summing up over the $\pi \nu_{\tau}$, $\rho \nu_{\tau}$ and $a_{1} \nu_{\tau}$ semileptonic decay channels for the other $\tau$ (this amounts to about $52 \%$ of the total decay rate).

Using the asymmetry (17) it is then possible to measure the AWMM. Collecting events from the decay of both taus, one gets a sensibility (within 1s.d.):

$$
\left|\operatorname{Re}\left(a_{\tau}^{w}\right)\right| \leq 4 \cdot 10^{-4}
$$

for the combined data for $\pi$ and $\rho$ channels.

Let us discuss the possibility of measuring this magnitude using the method outlined above. There, the analysis was done assuming vanishing absorptive parts in $a_{\tau}^{w}$. The real part of this magnitude appears in the transverse polarization. The actual calculation shows that this is not a very good approximation, and that both real and imaginary parts are of the same order of magnitude. We have shown that the best sensitivity one can expect is of the order of $10^{-4}$, so the result (5) for the standard weak-magnetic moment will not be accessible in such an experiment. In order to disentangle the $a_{\tau}^{w}$ term one has to subtract the tree level helicity-flip term coming from the Standard Model. This is the first term in the right hand side of Eq.(8) and Eq.(17). Then, if an anomalously large signal related to the observable persists, it should be attributed to physics beyond the Standard Model.

While the real part of the AWMM contributes to the transverse polarization, the absorptive part of the AWMM contributes to the CP-even terms of the normal polarization $Y_{+}$, and no mixing of this real and imaginary parts occurs in the polarization terms. The transverse polarization is then an observable related to the real part of the AWMM while the normal polarization (in the absence of a $\mathrm{CP}$-violating interaction) is a magnitude related to the absorptive part. The CP-even part of the normal polarization $Y_{+}$is given by Eq.(9). The analysis is then similar to the one made in Ref. 10 for the weak-electric form factor. There, the leading $d_{\tau}^{w}$ term is extracted from an asymmetry that picks up the $\sin \theta_{\tau} \cos \theta_{\tau} \sin \phi_{h}$ term. However, in this case it is much better (i.e. , the sensitivity 
is enhanced by a factor $3 \pi / 4$ ) to define an asymmetry that picks up the $\sin \phi_{h^{\mp}}$ term from $Y_{+}$:

$$
A_{s}^{\mp}=\frac{\int_{0}^{\pi} d \phi_{h}^{\mp} \frac{d \sigma}{d \phi_{h}^{\mp}}-\int_{\pi}^{2 \pi} d \phi_{h}^{\mp} \frac{d \sigma}{d \phi_{h}^{\mp}}}{\int_{0}^{\pi} d \phi_{h}^{\mp} \frac{d \sigma}{d \phi_{h}^{\mp}}+\int_{\pi}^{2 \pi} d \phi_{h}^{\mp} \frac{d \sigma}{d \phi_{h}^{\mp}}}
$$

After some algebra one finds

$$
A_{s}^{\mp}=\mp \alpha_{h} \frac{3 \pi \gamma}{4} c_{w} s_{w} \frac{v}{a^{2}} \operatorname{Im}\left(a_{\tau}^{w}\right)
$$

Under the same assumptions as for the real part, and collecting events for both negative and positive tau-decays, we obtain that for the pion channel (i.e. $h \equiv \pi)$ it is possible to put the following bounds for the absorptive part of $a_{\tau}^{w}$ :

$$
\left|\operatorname{Im}\left(a_{\tau}^{w}\right)\right| \leq 1.4 \times 10^{-3} \quad(\pi \text { channel })
$$

while for the $\rho$ channel we have

$$
\left|\operatorname{Im}\left(a_{\tau}^{w}\right)\right| \leq 2.0 \times 10^{-3} \quad \quad(\rho \text { channel })
$$

Combining these results one gets a sensibility:

$$
\left|\operatorname{Im}\left(a_{\tau}^{w}\right)\right| \leq 1.1 \times 10^{-3}
$$

We have shown that the best sensitivity one can expect in the measurement of these observables is of the order of $10^{-4}$, so the result (5) for the real and imaginary AWMM will not be accessible. Other proposals [9] have the same order of magnitude for the sensitivity to the real part of the AWMM $a_{\tau}^{w}$. Thus a measurement of the transverse and normal polarization of single taus offers an opportunity to put bounds on the weak moments induced by models beyond the standard theory.

\section{Acknowledgements}

G.A.G.S. thanks the Spanish Ministerio de Educación y Ciencia for a postdoctoral grant at the University of Valencia. This work has been supported in part by CICYT, under Grant AEN 93-0234, and by I.V.E.I.. 


\section{Appendix}

In this Appendix we explicitly show some of the formulas we have used in the computation of the AWMM.

Current conservation for a vector current $V^{\mu}$ implies that

$$
\left(p_{-}+p_{+}\right)_{\mu} \bar{u}\left(p_{-}\right) V^{\mu}\left(p_{-}, p_{+}\right) v\left(p_{+}\right)=0
$$

From the above equation we deduce that the form factor corresponding to $\left(p_{-}+p_{+}\right)^{\mu}$ should vanish. Furthermore, the Gordon identity

$$
\bar{u}\left(p_{-}\right) \gamma^{\mu} v\left(p_{+}\right)=\frac{1}{2 m} \bar{u}\left(p_{-}\right)\left[i \sigma^{\mu \nu}\left(p_{+}+p_{-}\right)_{\nu}+\left(p_{-}-p_{+}\right)^{\mu}\right] v\left(p_{+}\right)
$$

leaves only two independent vector form factors: the charge radius and the AWMM. When computing the loop integral in any of the diagrams of figure 1 or figure 2 , one naturally ends up with a vector and axial tensor structure constructed with the only vectors available, i.e. the external vectors $p_{-}$and $p_{+}$, and the vectors and axial vectors constructed entirely with the Gamma-matrices and the $\bar{u}$ and $v$ spinors. To extract the contribution to the AWMM one has to select the $\left(p_{-}-p_{+}\right)^{\mu}$ terms from the tensor structure of the loop integrals, in the basis of $\gamma^{\mu}$ and $\left(p_{-}-p_{+}\right)^{\mu}$ vectors.

We define the scalar, vector and tensor one-loop 3-point functions as:

$$
\begin{array}{r}
\left\{I_{00} ; I_{\mu} ; I_{\mu \nu}\right\}\left(p_{-}{ }^{2},\left(p_{-}+p_{+}\right)^{2}, p_{+}{ }^{2}, m_{A}{ }^{2}, m_{B}{ }^{2}, m_{C}{ }^{2}\right)=\frac{1}{i \pi^{2}} \times \\
\int d^{n} k \frac{\left\{1 ; k_{\mu} ; k_{\mu} k_{\nu}\right\}}{\left(k^{2}-m_{A}{ }^{2}\right)\left(\left(k-p_{-}\right)^{2}-m_{B}{ }^{2}\right)\left(\left(k+p_{+}\right)^{2}-m_{C}{ }^{2}\right)}
\end{array}
$$

These functions have the following Lorentz structure:

$$
\begin{aligned}
I^{\mu}= & \left(p_{-}-p_{+}\right)^{\mu} I_{10}+\left(p_{-}+p_{+}\right)^{\mu} I_{11} \\
I^{\mu \nu}= & \left(p_{+}{ }^{\mu} p_{+}{ }^{\nu}+p_{-}{ }^{\mu} p_{-}{ }^{\nu}\right) I_{21}+\left(p_{+}{ }^{\mu} p_{-}{ }^{\nu}+p_{-}{ }^{\mu} p_{+}{ }^{\nu}\right) I_{22}+ \\
& \left(p_{+}{ }^{\mu} p_{+}{ }^{\nu}-p_{-}{ }^{\mu} p_{-}{ }^{\nu}\right) I_{2-1}+\left({p_{+}}^{\mu} p_{-}{ }^{\nu}-p_{-}{ }^{\mu} p_{+}{ }^{\nu}\right) I_{2-2}+g^{\mu \nu} I_{20}
\end{aligned}
$$

To extract the contribution to the AWMM one only needs to calculate the $I_{00}, I_{10}, I_{21}$

and $I_{22}$ type integrals. Current conservation and the structure of the contractions of 
the $I_{\mu \nu}$ integrals that appears in the calculus (i.e., $I_{\eta \nu} g^{\eta \nu} \gamma^{\mu}$ and $I_{\mu \nu} \gamma^{\nu}$ ) eliminates $I_{11}$, $I_{2-1}, I_{2-2}$ and $I_{20}$. These functions can be written in terms of analytic and dilogarithm functions, as shown in [18]. Furthermore, one can also express all of them in terms of the $I_{00}$ function and the 2-point functions. To compute the actual value of the AWMM we have explicitly done the calculus of these functions for the external momenta on-shell and for the different masses circulating in the loop, and we have checked these results in the $m_{\tau} \rightarrow 0$ limit. In the following we list the results for the $I_{i j}$ functions (in the $m_{\tau} \rightarrow 0$ limit) needed for the $a_{A B C}$ contributions given in Eq.(3)), except for $a_{\gamma \tau \tau}$ that it is proportional to the well known result for the anomalous magnetic moment at $q^{2}=M_{Z}^{2}$. As all external lines are on the mass shell, the arguments of the $I_{i j}$ are given by the square of the masses of the particles in the corresponding diagram. For $a_{z \tau \tau}$ they are:

$$
\begin{aligned}
& I_{00}\left(m_{\tau}^{2}, M_{Z}^{2}, m_{\tau}^{2}, M_{Z}^{2}, 0,0\right)=\frac{1}{M_{Z}^{2}}\left(-\frac{\pi^{2}}{12}-i \pi \ln 2\right) \\
& I_{10}\left(m_{\tau}^{2}, M_{Z}^{2}, m_{\tau}^{2}, M_{Z}^{2}, 0,0\right)=\frac{1}{M_{Z}^{2}}\left(-1+\frac{\pi^{2}}{12}+i \pi(\ln 2-1)\right) \\
& I_{21}\left(m_{\tau}^{2}, M_{Z}^{2}, m_{\tau}^{2}, M_{Z}^{2}, 0,0\right)=\frac{1}{M_{Z}^{2}}\left(\frac{3}{4}-\frac{\pi^{2}}{12}+i \pi\left(\frac{1}{2}-\ln 2\right)\right) \\
& I_{22}\left(m_{\tau}^{2}, M_{Z}^{2}, m_{\tau}^{2}, M_{Z}^{2}, 0,0\right)=\frac{1}{M_{Z}^{2}}\left(-\frac{5}{2}+\frac{\pi^{2}}{4}-i \pi(2-3 \ln 3)\right)
\end{aligned}
$$

For $a_{\nu W W}, a_{\nu W \sigma}$ and $a_{\nu \sigma W}$, we define $r=\frac{M_{W}^{2}}{M_{Z}^{2}}$, and they are:

$$
\begin{gathered}
I_{00}\left(m_{\tau}^{2}, M_{Z}^{2}, m_{\tau}^{2}, 0, M_{W}^{2}, M_{W}^{2}\right)=\frac{1}{M_{Z}^{2}}\left(L i_{2}\left(\frac{r}{r-r_{+}}\right)-L i_{2}\left(\frac{r-1}{r-r_{+}}\right)+\right. \\
\left.L i_{2}\left(\frac{r}{r-r_{-}}\right)-L i_{2}\left(\frac{r-1}{r-r_{-}}\right)+L i_{2}(r)+\ln (r) \ln (1-r)-\frac{1}{2} \ln ^{2}(r)\right) \\
I_{10}\left(m_{\tau}^{2}, M_{Z}^{2}, m_{\tau}^{2}, 0, M_{W}^{2}, M_{W}^{2}\right)=\frac{1}{M_{Z}^{2}}\left(2 \sqrt{4 r-1} \tan ^{-1}\left(\frac{1}{\sqrt{4 r-1}}\right)-1\right)+r I_{00} \\
I_{21}\left(m_{\tau}^{2}, M_{Z}^{2}, m_{\tau}^{2}, 0, M_{W}^{2}, M_{W}^{2}\right)=\frac{1}{M_{Z}^{2}}\left(-\frac{3}{4}-r+(1+2 r) \sqrt{4 r-1} \times\right. \\
\left.\tan ^{-1}\left(\frac{1}{\sqrt{4 r-1}}\right)\right)+r^{2} I_{00}
\end{gathered}
$$




$$
I_{22}\left(m_{\tau}^{2}, M_{Z}^{2}, m_{\tau}^{2}, 0, M_{W}^{2}, M_{W}^{2}\right)=-\frac{1}{2} \frac{1}{M_{Z}^{2}}-2 r I_{10}
$$

where $r_{ \pm}=\frac{1 \pm \sqrt{1-4 r}}{2}$ and $L i_{2}$ is the dilogarithm function. For $a_{\tau \Phi Z}$ and $a_{\tau Z \Phi}$, when $M_{\Phi}=2 M_{Z}$, they are:

$$
\begin{aligned}
I_{10}\left(m_{\tau}^{2}, M_{Z}^{2}, m_{\tau}^{2}, 0, M_{Z}^{2}, M_{\Phi}^{2}\right)= & \frac{1}{M_{Z}^{2}} \frac{1}{2}\left(-2-\frac{2}{3} \pi^{2}+6 \ln 2-4 i \pi \ln \frac{3}{4}-\ln ^{2} 2+\right. \\
& \left.4 L i_{2}\left(\frac{3}{4}\right)+8 L i_{2}(2)-8 L i_{2}\left(\frac{3}{2}\right)\right)
\end{aligned}
$$

For $a_{W \nu \nu}$, the functions are:

$$
\begin{aligned}
I_{00}\left(m_{\tau}^{2}, M_{Z}^{2}, m_{\tau}^{2}, M_{W}^{2}, 0,0\right)=\frac{1}{M_{Z}^{2}}\left(-(i \pi+\ln (r)) \ln \left(1+\frac{1}{r}\right)+L i_{2}\left(-\frac{1}{r}\right)\right) \\
I_{10}\left(m_{\tau}^{2}, M_{Z}^{2}, m_{\tau}^{2}, M_{W}^{2}, 0,0\right)=\frac{1}{M_{Z}^{2}}\left(-1-\ln (r)-i \pi+(i \pi+\ln (r)) r \ln \left(1+\frac{1}{r}\right)-\right. \\
\left.r L i_{2}\left(-\frac{1}{r}\right)\right) \\
I_{21}\left(m_{\tau}^{2}, M_{Z}^{2}, m_{\tau}^{2}, M_{W}^{2}, 0,0\right)=\frac{1}{M_{Z}^{2}}\left(-\frac{1}{4}+r+\left(r-\frac{1}{2}\right) \ln (r)+i \pi\left(r-\frac{1}{2}\right)-\right. \\
\left.(i \pi+\ln (r)) r^{2} \ln \left(1+\frac{1}{r}\right)+r^{2} L i_{2}\left(-\frac{1}{r}\right)\right) \\
I_{22}\left(m_{\tau}^{2}, M_{Z}^{2}, m_{\tau}^{2}, M_{W}^{2}, 0,0\right)=\frac{1}{M_{Z}^{2}}\left(\frac{1}{2}+2 r+2 r \ln (r)+2 i r^{2} \pi+(1+2 r) r \times\right. \\
\left.\left(\operatorname{Lin}\left(-\frac{1}{r}\right)-(i \pi+\ln (r)) \ln \left(1+\frac{1}{r}\right)\right)\right) \quad(\mathrm{A} .9)
\end{aligned}
$$

\section{References}

[1] D.Decamp et al., ALEPH Collab., Phys. Lett. B 265 (1991) 430; G.Alexander et al., OPAL Collab., Phys. Lett. B266 (1991) 201; P.Abreu et al., DELPHI Collab., Zeit. für Physik C55 (1992) 555; O.Adriani et al., L3 Collab., Phys. Lett. B294 (1992) 466.

[2] S.Jadach et al. in: Z.Physics at LEP 1, eds. G.Altarelli, R.Kleiss and C.Verzegnassi, CERN Report CERN 89-08,Vol. 1, p.235. 
[3] R.Alemany et al., Nucl. Phys. B379 (1992) 3.

[4] P.Overmann, Dortmund University preprint DO-TH 93/24 (1993).

[5] K.Abe et al., Phys. Rev. D73 (191994) 25.

[6] W.Bernreuther and O.Nachtmann, Phys. Rev. Lett. 63 (1989) 2787 and erratum Phys. Rev. Lett. 64 (1990) 1072.

[7] W.Bernreuther et al., Zeit. für Physik C52 (1991) 567.

[8] W.Bernreuther, O.Nachtmann and P.Overmann, Phys. Rev. D48 (1993) 78.

[9] U.Stiegler, Zeit. für Physik C57 (1993) 511.

[10] J.Bernabéu, G.A.González-Sprinberg and J.Vidal, Phys. Lett. B326 (1994) 168.

[11] Y.S.Tsai, Phys. Rev. D4 (1971) 2821 and erratum Phys. Rev. D13 (1976) 771.

[12] J.H.Kühn and F.Wagner, Nucl. Phys. B236 (1984) 16.

[13] A.Rougé, Zeit. für Physik C48 (1990) 75.

[14] K.Hagiwara, A.D.Martin and D.Zeppenfeld, Phys. Lett. B235 (1990) 198.

[15] K.Fujikawa, B.W.Lee and A.I.Sanda, Phys. Rev. D6 (1972) 2923.

[16] A.Queijeiro, Zeit. für Physik C60 (1993) 667.

[17] J.H.Kühn, Phys. Lett. B313 (1993) 458.

[18] G.t'Hooft and M.Veltman, Nucl. Phys. B153 (1979) 365; G.Passarino and M. Veltman, Nucl. Phys. B160 (1979) 151. 